\title{
Functional outcome after symtomatic internal carotid artery occlusion
}

\author{
Ioana Stanescu' ${ }^{12}$, Angelo Bulboaca ${ }^{2}$, Dana Fodor', Gabriela Dogaru ${ }^{12}$ \\ Corresponding author: Ioana Stanescu, E-mail: ioanastane@yahoo.com
}

\section{Abstract}

Internal carotid artery occlusion accounts for $15-20 \%$ of ischemic strokes, caused by atherosclerosis or dissection. Clinical symptoms are variable, from asymptomatic cases to minor or severe strokes. Diagnosis in internal carotid artery (ICA) occlusion is based on imaging techniques. Prognosis after ICA occlusion depends on many factors: severity of neurologic deficit, spontaneous recanalization of the artery, and the occurrence of recurrent strokes. Patients with spontaneous recanalization of the occluded ICA tend to have a retained functional ability and favorable clinical outcomes. Medical treatment, recanalization techniques and intensive rehabilitation program are essential in improving functional outcome of patients with stroke produced by ICA occlusion. We present the case of a young patient diagnosed with ischemic stroke produced by internal carotid artery occlusion, with consecutive severe neurologic deficit, and an unfavorable functional outcome, as evaluated with the Modified Rankin Scale. Spontaneous recanalization of the occluded ICA was observed after 6 weeks, suggesting a carotid dissection. The patient was included in an intensive rehabilitation program, associated with best medical therapy, showing improvement of its functional status at 3 months follow up.

Key words: ischemic stroke, carotid artery occlusion, carotid dissection, spontaneous recanalization, functional outcome,

\section{Introduction}

Internal carotid artery (ICA) occlusion is a frequent cause of ischemic stroke. In general population, the real prevalence and incidence of ICA occlusion is difficult to assess, because many patients remain asymptomatic or are not investigated (1).

ICA occlusion is produced by atherosclerotic disease, cardioembolism, dissection or radio-therapy $(2,3)$. Clinical symptoms are variable, from asymptomatic cases to minor or severe strokes. Diagnosis in symptomatic ICA occlusion is based on one of the following imaging techniques, used concurrently: Doppler ultrasound examination of cervical arteries, computer tomograph angiography (CTA), magnetic resonance angiography (MRA) and digital substraction arteriography (DSA) (2).

Asymptomatic patients with ICA occlusion have a favourable outcome, but symptomatic ICA occlusions are associated with important disabilities and carried also an important risk of recurrent strokes. Spontaneous recanalization of the occluded ICA is not a rare event, it has been described in up to $10 \%$ of the patients, especially in ICA dissection with secondary occlusion, and in the first weeks after

stroke (3, 4, 5). Patients with late spontaneous recanalization of the occluded ICA tend to have a retained functional ability and favorable clinical outcomes (6).

Treatment options in ischemic strokes caused by ICA occlusion are not different of standard ischemic stroke treatment in the acute phase. In chronic phase, best medical treatment combined with surgical or endovascular recanalization techniques could help in obtaining a functional improvement.

\section{Case report:}

A 45 years old male patient, smoker, with moderate alcohol consumption, was admitted in the Emergency Department for severe left hemiplegia. Mild right hemicrania was then reported. Emergency native CT scan showed acute infarctus in the right middle cerebral artery (MCA) territory (images not shown). Intravenous thrombolysis was not performed, due to unknown onset time. Doppler ultrasound of cervical arteries showed complete occlusion of the right ICA. Blood pressure values were normal. Cardiologic assessment (including transthoracic echocardiography and 24 hours Holter-ECG) was unremarkable. 
Blood tests revealed slightly elevated cholesterol and triglyceride levels. The patient was discharged on antiplatelet (clopidogrel) and statin treatment.

Six weeks after the acute stroke, he was admitted in our Rehabilitation hospital. He reported intermittent headache exacerbation. Neurological assessment showed severe left hemiparesis with brachial predominance (muscle force quotation of $1 / 5$ in left upper limb and $3 / 5$ in left lower limb), left hypoesthesia, and severely impaired gait requiring bilateral assistance. Functional assessment showed a modified Rankin Scale (mRS) of 4, the patient being completely dependent in his daily activities. Native CT scan showed large chronic ischemic area in the right MCA territory (Figure 1).
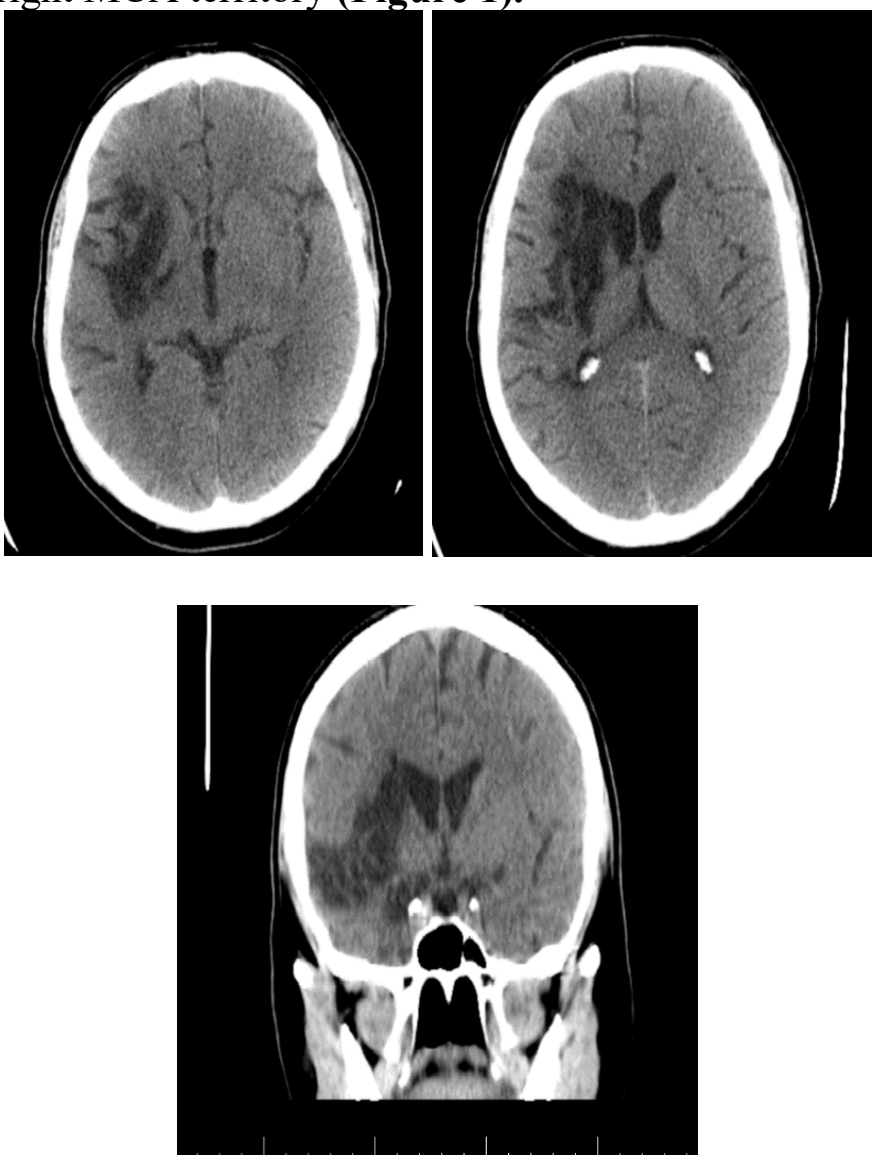

Fig. 1: Native CT scan 6 weeks after stroke onset showing chronic ischemic area in the territory of the right MCA

Doppler ultrasound of cervical arteries was repeated, and showed eccentric flow in the right ICA. CTA was then performed, and confirmed recanalization of the right ICA (Figures 2 and 3).
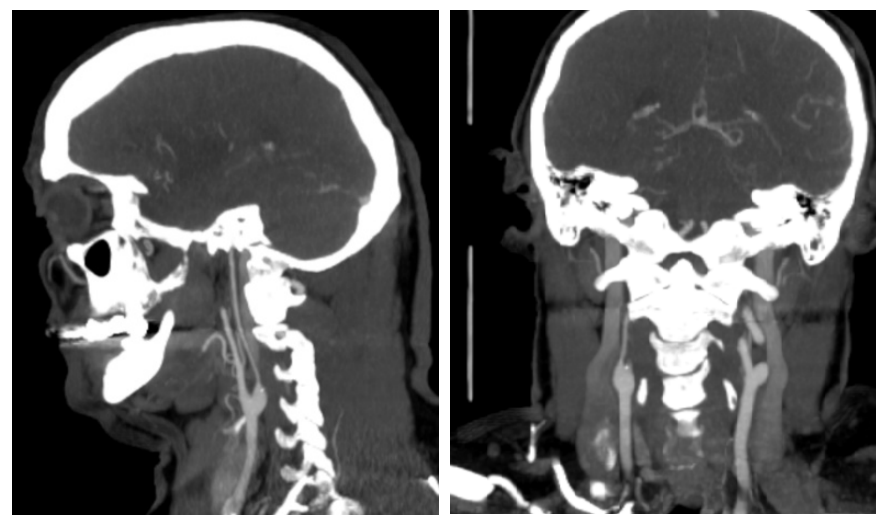

Fig. 2: Recanalization of the extracranial right ICA from origin to intracranial segments, suggestive for dissection (right- lateral view, left - coronal view)
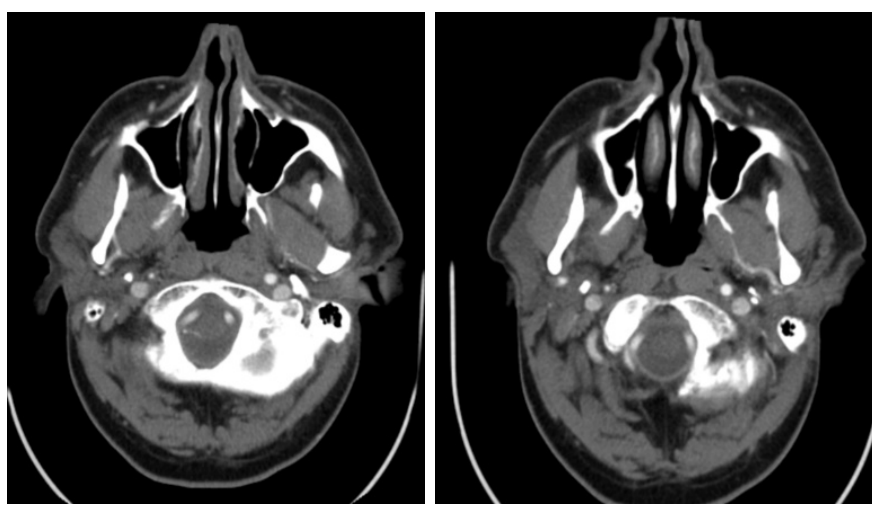

Fig. 3: Recanalization of intracranial segment of right ICA

The length of previous stenosis, with early recanalization, which extends intracranially, suggest an ICA dissection as the cause of ICA occlusion. Conservative medical treatment was chosen for our patient, carotid artery dissections have generally a good prognosis. The patient was discharged on antiplatelet (clopidogrel) and statin treatment. Control Doppler ultrasound was settled after 3 months.

The patient was admitted in an intensive rehabilitation program. The physical therapy methods consisted in sedative massage of the dorsal and lumbar paravertebral muscles, postural exercises and passive mobilization of the paralyzed limbs to prevent tendinous retractions and respiratory exercises. For upper limb function, task-specific motor training, oriented towards achieving daily activity goals, and virtual therapy were used. Gait training included assisted walking on the parallel bar system. Robotic devices were used for improvement of upper and lower limb functions. 
For lower limbs, intelligent control of walking was provided by a robotic system, which allowed partial support of patient's body weight with walking assistance depending of patient's muscle force and movement capacity. For upper limb, a prototype of exoskeleton device focused of elbow movement was used (7). The rehabilitation program was conducted over three weeks, with mild improvement of motor function.

At 3 months follow-up, patient neurological status improved: muscle force was $3 / 5$ proximally in left upper limb, and 4/5 in right lower limb, the gait was possible with unilateral assistance, and patient regained partial autonomy, with functional improvement and a modified Rankin score of 3 points (8). Doppler ultrasound of cervical arteries confirmed ICA recanalization with high velocity flow.

\section{Results and discussions}

Stenosis of extracranial arteries accounts for $15-20 \%$ of all ischemic strokes in populational studies $(9,10$, 11), while extracranial ICA occlusion is responsible for $3,5 \%$ (12) reported incidence of ICA occlusion being 6/100 000 individuals (11).

Factors involved in ICA occlusion are atherosclerotic disease, dissection of the extracranial carotid artery, cardioembolism and neck radiotherapy $(2,3)$. In older persons, ICA occlusion could result from a progression of a tight carotid stenosis (13). Dissection with secondary ICA occlusion is more common in young, and accounts for $2,5 \%$ of ischemic strokes (14). Elevated homocysteine levels were also associated with ICA occlusion (15).

In our 45 years old male patient, without main atherosclerosis risk factors, the presumed etiology for ICA occlusion was arterial dissection. Diagnosis of ICA occlusion in our stroke patient relayed on Doppler ultrasound of cervical arteries, CTA and cervical MRA sequences for dissection diagnosis were not performed.

The clinical presentation after an acute ICA occlusion is variable: some persons remain asymptomatic, others have transient ischemic attacks (TIA) or minor strokes, and others have severe strokes (2). The severity of neurologic deficit depends on the presence of collateral circulation and on early recanalization of the artery (16). A study published in 1986 found severe neurologic deficits in all patients with ICA occlusion (17). In our patient, the severity of neurologic deficit could be explained by brutal onset, lack of collateral circulation or a dysfunctional Willis polygon.

Asymptomatic ICA occlusion has a usually a benign evolution, when the collateral cerebral circulation is well-developed and the occluded ICA remains stable ("safe artery") (18). The natural history of symptomatic ICA occlusion carries a risk of recurrent stroke of $5-6 \%$ per year (2). A new stroke after a complete ICA occlusion is explained by "carotid stump syndrome" (microemboli from the stump or from ipsilateral external carotid artery occluding middle cerebral artery branches) or by hypoperfusion infarcts in watershed territories (in case of insufficient compensation by collateral circulation) (2). This is the reason why symptomatic ICA occlusion patients required best medical or/and surgical treatments.

Our patient was treated with antiplatelet agents and high-dose statins; no recurrence of ischemia and spontaneous recanalization were observed after 6 months form stroke onset. Diagnosis of spontaneous recanalization was established with Doppler ultrasound, and confirmed with CTA, where the recanalized ICA showed small, irregular diameter which extends from the origin until the beginning of the petrous segment, the shape and length being suggestive of dissection.

Spontaneous recanalization of occluded ICA has variable rates: between $2,3 \%$ and $10,3 \%$ (4), and is most likely to occur in the acute phase (in the first 2 weeks after occlusion) (19), but was also described in chronic ICA occlusion, after more than 3 months from onset, being considered a rare event (20). Spontaneous recanalization increases the cerebral blood flow in the affected brain area and the risk of subsequent embolic stroke. In occlusions produced by ICA dissection, spontaneous recanalization is more frequent, in 57 to $62 \%$ of cases (5), appeared earlier, between 2 days and 6 weeks after stroke, and increased over time (3). The recanalization of an occluded atherosclerotic ICA seems to be a rare event. Mechanisms of late spontaneous recanalization are partially unknown: fibrinolysis and collateral circulation through vasa vasorum (6).

Treatment of ischemic stroke produced by ICA occlusion is not different from treatment of any ischemic stroke, but the prognosis after intravenous thrombolysis treatment seems to be unfavorable at 3 months (21).

The natural history of strokes induced by ICA occlusion showed good recovery in only $2-12 \%$ of 
patients and important disability in $40-69 \%$ of patients $(17,22)$, as measured by modified Rankin Scale (mRS) (8). Unfavorable outcome with $75 \%$ rate of death and disability after 1,2 years of follow up have also been reported in ICA occlusion strokes (23).

In our patient, despite severe hemiplegia induced by stroke, evolution was favorable, with improvement of motor deficit and disability, possibly related to spontaneous recanalization of the occluded right ICA and to intensive rehabilitation program.

Treatment options for chronic extracranial ICA occlusion include best medical treatment (with oral antiplatelet drugs: aspirin or combination of aspirin with clopidorel) - aiming to prevent further strokes, and recanalization therapy: carotid endarterectomy (CEA), stenting (CAS), or hybrid surgery (CEA of the proximal part of ICA and stenting of the distal part) (2). Indications for surgical treatment are still under debate: symptomatic patients, with severe cerebral hemodynamic failure (as measured by PET as increased oxygen extraction extraction fraction OEF) and retrograde filling of ICA are good candidates for surgical recanalization. Recanalization has a succes rate of only $34 \%(24,25)$. For our patient, treatment options included only best medical treatment and rehabilitation. No recurrent strokes were noted on follow up, and patient's functional outcome tends to improve.

\section{Conclusions}

Occlusion of internal carotid artery accounts for up to $20 \%$ of ischemic strokes. In young people, carotid dissections leading to ICA occlusion are a frequent cause of stroke. Diagnosis relays on concurrent imaging methods. Clinical picture of these particular strokes depends on patient's cerebral hemodynamic particularities and on the possibility of spontaneous recanalization of the occluded artery.

Despite severity of neurological status, spontaneous recanalization could help in improving disability in combination with intensive rehabilitation program, while medical treatment is essential in preventing stroke recurrences.

Endovascular or surgical recanalization of occluded artery is recommended if previous methods have failed in improving functional outcome of patients.

\section{Conflict of interest}

There is no conflict of interest for any of the authors regarding this paper.

\section{Informed consent}

An informed consent was obtained from the patient included in this study.

\section{References}

1. Thanvi B, Robinson T. Complete occlusion of extracranial internal carotid artery: clinical features, pathophysiology, diagnosis and management. Postgrad Med J. 2007;83(976):9599.

2. Xu B, Li C, Guo Y, Xu K, Yang Y, Yu J. Current understanding of chronic total occlusion of the internal carotid artery. Biomed Rep. 2017;8(2):117-125. doi:10.3892/br.2017.1033.

3. Lanari A, Silvestrelli G. Acute and Chronic Carotid Occlusion Syndromes. In Paciaroni M, Agnelli G, Caso V, Bogousslavsky J (eds): Manifestations of Stroke. Front Neurol Neurosci. Basel, Karger. 2012, vol 30, pp 185-190.

4. Morris-Stiff G, Teli M, Khan PY, Ogunbiyi SO, Champ CS, Hibberd R, Brown R, Bailey DM, Winter RK, Lewis MH. Internal carotid artery occlusion: Its natural history including recanalization and subsequent neurological events. Vasc Endovascular Surg. 2013;47:603607.

5. Eui Jong K, Jun Seok K, Woo Suk C. Carotid artery stenting in a patient with spontaneous recanalization of a proximal internal carotid artery occlusion: a case report. Korean J Radiol. 2006; 7: 292-6.

6. Wu H, Liu Y, Li B, Zhang H, Liu C. Prognosis of patients with late spontaneous recanalization of the atherosclerotic occlusion of internal carotid arteries: A pilot case series. Exp Ther Med. 2018 Nov;16(5):4271-4276.

7. Bulboacă AE, Bolboacă SD, Bulboacă AC. Ethical considerations in providing an upper limb exoskeleton device for stroke patients. Medical hypotheses. 2017;101: 61-64.

8. Quinn TJ, Lees KR, Hardemark HG, Dawson J, Walters MR. Initial experience of a digital training resource for modified Rankin scale assessment in clinical trials. Stroke. 2007;38:2257-2261. 
9. Petty GW, Brown RD Jr, Whisnant JP, Sicks JD, O'Fallon WM, Wiebers DO. Ischemic stroke subtypes: a population-based study of incidence and risk factors. Stroke.1999 Dec;30(12):2513-6.

10. Schneider AT, Kissela B, Woo D, et al. Ischemic stroke subtypes: A population-based study of incidence rates among blacks and whites. Stroke. 2004;35:1552-1556.

11. Flaherty M L, Flemming K D, McClelland R. et al. Population-based study of symptomatic internal carotid artery occlusion. Incidence and long-term follow-up. Stroke 200435e349ed .

12. Flaherty ML, Kissela B, Khoury JC, Alwell K, Moomaw CJ, Woo D, Khatri P, Ferioli S, Adeoye O, Broderick JP, Kleindorfer D. Carotid artery stenosis as a cause of stroke. Neuroepidemiology. 2013;40(1):36-41.

13. den Hartog AG, Halliday AW, Hayter E, Pan H, Kong X, Moll FL, de Borst GI. Risk of Stroke From New Carotid Artery Occlusion in the Asymptomatic Carotid Surgery Trial-1. Stroke. 2013;44:1652-1659.

14. Bogousslavsky J, Despland PA, Regli F. Spontaneous carotid dissection with acute stroke. Arch Neurol. 1987 Feb;44(2):137-40.

15. Jeong SK, Seo JY, Cho YI. Homocysteine and internal carotid artery occlusion in ischemic stroke. J Atheroscler Thromb. 2010 Sep 30;17(9):963-9.

16. Lee JI, Jander S, Oberhuber A, Schelzig H, Hanggi D, Turowski B, Seitz RJ. Stroke in patients with occlusion of the internal carotid artery: options for treatment. Expert Rev. Neurother. Early online, 1-15 (2014).

17. Meyer FB, Sundt TM, Jr, Piepgras DG, et al. Emergency carotid endarterectomy for patients with acute carotid occlusion and profound neurological deficits. Ann Surg. 1986;203:8289.
18. Powers WJ, Derdeyn CP, Fritsch SM, Carpenter DA, Yundt KD, Videen TO, Grubb RL Jr. Benign prognosis of never-symptomatic carotid occlusion. Neurology. 2000 Feb 22;54(4):87882.

19. Nguyen-Huynh MN, Lev MH, Rordorf G. Spontaneous recanalization of internal carotid artery occlusion. Stroke 2003;34: 1032-4.

20. Delgado MG, Vega PP, Lahoz CH, Calleja S. Late spontaneous recanalization of symptomatic atheromatous internal carotid artery occlusion. Vascular. 2015;23:211-216.

21. Paciaroni M, Agnelli G, Caso V, et al. Intravenous thrombolysis for acute ischemic stroke associated to extracranial internal carotid artery occlusion: the ICARO-2 study. Cerebrovasc Dis 2012; 34(5-6):430-5.

22. Li W, Yin Q, Xu G, Liu X. Treatment Strategies for Acute Ischemic Stroke Caused by Carotid Artery Occlusion. Interv Neurol. 2016;5(34):148-156.

23. Paciaroni M, Caso V, Venti M, Milia P, Kappelle L, J, Silvestrelli G, Palmerini F, Acciarresi M, Sebastianelli M, Agnelli G: Outcome in Patients with Stroke Associated with Internal Carotid Artery Occlusion. Cerebrovasc Dis. 2005;20:108-113.

24. Chen YH, Leong WS, Lin MS, Huang CC, Hung CS, Li HY, Chan KK, Yeh CF, Chiu MJ, Kao HL. Predictors for Successful Endovascular Intervention in Chronic Carotid Artery Total Occlusion. JACC Cardiovasc Interv. 2016;9:1825-1832.

25. Camporese G, Labropoulos N, Verlato F, Bernardi E, Ragazzi R, Salmistraro G, Kontothanassis D, Andreozzi GM. Carotid Recanalization Investigators Group: Benign outcome of objectively proven spontaneous recanalization of internal carotid artery occlusion. J Vasc Surg. 2011;53:323-329. 